\title{
STUDY OF GENERIC FRONT-END DESIGNS FOR ERL BASED LIGHT SOURCES
}

\author{
Guimei Wang ${ }^{\mathrm{a}, \mathrm{b}}$, Yu-Chiu Chao ${ }^{\mathrm{a},+}$, George Neil ${ }^{\mathrm{a}}$, Pavel Evtushenko ${ }^{\mathrm{a}}$, Kui Zhao ${ }^{\mathrm{b}}, \mathrm{Xiangyang}_{\mathrm{Lu}}^{\mathrm{b}}{ }^{\mathrm{b}}$, \\ Jiejia Zhuang ${ }^{\mathrm{b}}$, Chuyu Liu ${ }^{\mathrm{b}}$, Jiaer Chen ${ }^{\mathrm{b}}$, \\ ${ }^{\mathrm{a}}$ Jefferson Lab., Newport News, VA, USA \\ ${ }^{\mathrm{b}}$ Inst. of Heavy Ion Physics \& MOE lab. of Heavy Ion Physics, Peking University, Beijing, China
}

\section{Abstract}

We present work directed at examining the performance of various front end components of an ERL based light source. These include electron source, bunch compression, merger, and accelerating sections, with parameter space dictated by proposed facilities (at FSU and Peking University). These facilities share enough common structural features to make the study applicable to both to a large extent. In this report we will discuss the $6 \mathrm{D}$ phase space evolution through the front end based on simulation, with reliable modeling of magnetic and superconducting RF fields. Discussion will be devoted to relative merits of alternative designs, robustness and operational scenarios.

\section{INTRODUCTION}

With the development of superconducting linear accelerator and electron sources, energy recovery linac (ERL) technology makes it possible to generate high repetition rate and low emittance electron beam. Especially after successful experimental results in Jefferson Lab FEL, JAEA FEL and Novosibirsk FEL, ERL is proved to get high power beam at low energy and is supposed to extend high energy for synchrotron light source for its high brightness merit, compared with storage ring.

In the front end, from injector to the accelerator, usually composed of matching quads and merger, the energy of electron beam is low and high order term and space charge effect are the main effects that will deteriorate beam emittance.

In this paper, DC voltage, RF field gradient and RF phase and space charge force are optimized. Different merger structure is studied in error sensitivity. Also bunch compression in the merger is discussed.

\section{INJECTOR OPTIMIZATION}

Peking University(PKU) is upgrading injector from $1+1 / 2$ cell to $3+1 / 2$ cell DC-SC photo injector ${ }^{[1]}$.In injector, radial electrostatic in DC gun, electromagnet field in cavity and space charge together affect the emittance of electron beam. The radial electrostatic field, at the surface of cathode, is focusing and is defocusing at the beam pipe. $\mathrm{RF}$ field has focusing force. It is directly decided by field gradient and RF phase. But transverse space charge

*Work supported by National 973 Projects and U.S. DOE

Contract No. DE-AC05-06OR23177

\#wangguimei@pku.edu.cn,+chao@jlab.org always has defocusing force and it is proportional to bunch charge. These three factors together give different beam distribution and will affect the emittance in the match section and merger section.
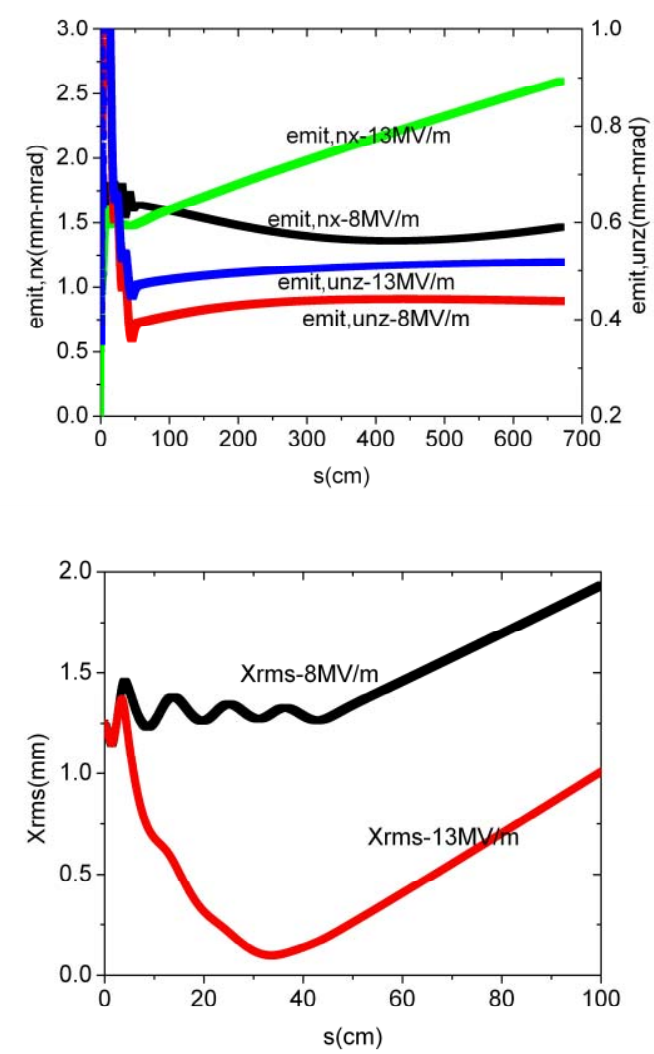

Figure 1a: Beam emittance and beam size along beam line

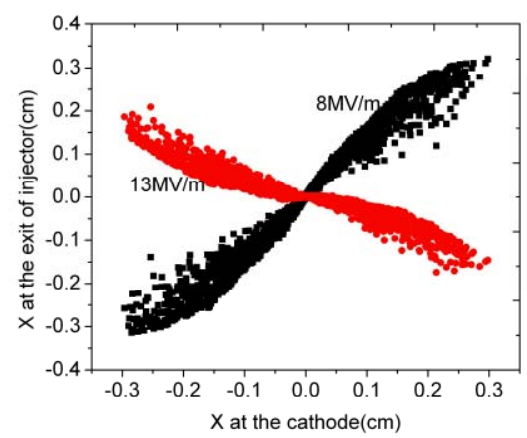

Figure.1b: Coordinate $\mathrm{x}$ at injector exit vs $\mathrm{x}$ at cathode. 
With the code PARMELA, for $20 \mathrm{pC}$ bunch charge, Fig.1a shows the emittance from cathode to $6.4 \mathrm{~m}$ drift space(almost the same length as match and merger) after injector for different average axial electric field gradient at $8 \mathrm{MV} / \mathrm{m}$ and $13 \mathrm{MV} / \mathrm{m}$ and Fig. $1 \mathrm{~b}$ shows the particle transverse coordinate at the exit of injector(at $59 \mathrm{~cm}$ ) VS the coordinate at the cathode. As the injector is cylindrically symmetric along $\mathrm{Z}$, so here just shows $\mathrm{X}$ as transverse. From Fig.1a, we can see that, after the injector, for different Eacc, the longitudinal unnormalized emittance almost has the same trend, but the transverse normalized emittance has big difference. For $8 \mathrm{MV} / \mathrm{m}$, the transverse emittance decrease at first, then increase, while for $13 \mathrm{MV} / \mathrm{m}$, it increase greatly and simulation also shows that it can not be compensated with solenoid. It can be explained that the RF focusing inside the cavity works similar to solenoid and for different Eacc, it works in two different region crossover and waist as defined in reference [2]. Fig.1b confirms this explanation. Fig.1a also shows that the beam size inside the cavity for $13 \mathrm{MV} / \mathrm{m}$ has a very small waist, which means the space charge force around waist is very strong. The result is confirmed by ASTRA.
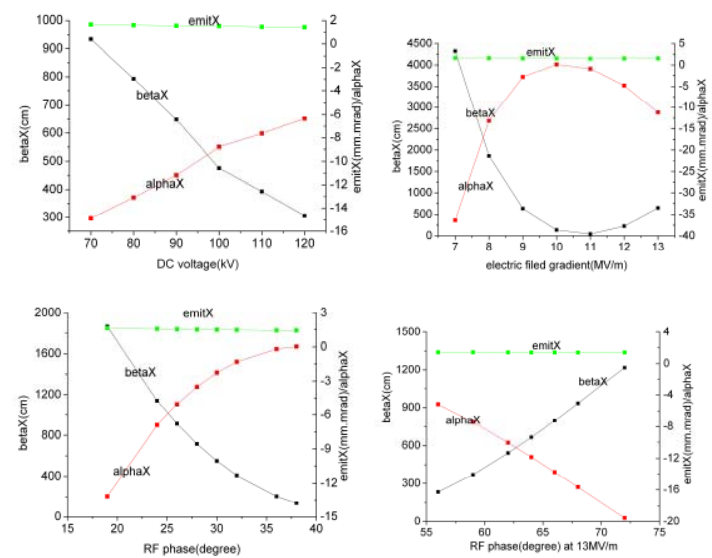

Figure.2: DC, electric field gradient scan and RF phase.

To control the emittance growth after injector, the main factors, DC voltage, Eacc and RF phase are studied. Fig. 2 gives betaX, alphaX, and normalized transverse emittance emitX along with DC voltage from $70 \mathrm{kV}$ to $120 \mathrm{kV}$, Eacc from $7 \mathrm{MV} / \mathrm{m}$ to $13 \mathrm{MV} / \mathrm{m}$ and $\mathrm{RF}$ phase scan from \pm 10 degree around crest. Looking at the four graphs in Fig. 2, in the upper left we see the response to $\mathrm{DC}$ voltage. In the upper right is the response to RF field gradient. The lower two graphs show response to RF phase at $8 \mathrm{MV} / \mathrm{m}$ and $13 \mathrm{MV} / \mathrm{m}$, respectively. Combining with the Fig.1, we know on right curve, it is the crossover region and transverse emittance will grow. So we get the conclusion that RF average axial electric field will change beam property dramatically and DC voltage and RF phase affect but not fundamentally beam property. The best Eacc for $20 \mathrm{pC}$ is about $10 \mathrm{MV} / \mathrm{m}$. The longitudinal beam property is simulated to be mainly affected by RF phase. It is reasonable and consistent with Fig.1. In the cavity, beam has two forces, transverse space charge force and
$\mathrm{RF}$ force. Transverse space charge force is proportional to bunch charge and RF force is proportional to Eacc. It can be expected with bunch charge increases, the optimized Eacc will increase, which is also confirmed for $300 \mathrm{pC}$ in ref[1].

\section{DIFFERENT MERGER STRUCTURE}

Merger is used to transport beam from injector as well as recirculated beam to accelerator. The two beams have different energy and come from different beam line. There are usually four different achromatic merger structures, three dipole merger in JLAB FEL, chicane structure in CEBAF or BINP, dogleg structure in JAEA and zigzag structure proposed from BNL.

In the merger, the beam energy from injector is relatively low. So space charge effect ${ }^{[3]}$ is one factor that will cause emittance growth. Also the field error, which makes the merger deviate from the nominal achromatic design, induces emittance growth. Here, DIMAD is used to analyze central beam orbit $\mathrm{x}$ (which is bend plane) and $y$ for different energy spread $\pm 1 \%$ in Fig. 3 . In these merger structures, the drift space between the first and second bend is the same and the bend angle is 20degree, except zigzag, which has 10degree and 20degree. There is $1 \mathrm{~m}$ drift space after the last bend, which is useful to see the error trend. Fig. 3 shows that the orbit displacement at the last bend in zigzag and chicane is about half of displacement in dogleg and three dipole merger. But after $1 \mathrm{~m}$ drift, it shows great difference. For y plane in zigzag and $\mathrm{x}$ and $\mathrm{y}$ plane in chicane, the displacement for different energy spread is distributed on \pm 0 and it is almost kept small after drift. While for the rest cases, the displacement for different energy spread is just larger or smaller than 0 , and it is amplified in drift space, which indicates the emittance will grow in this case in drift space. On the other hand, the displacement is relatively small at cases of zigzag y plane, dogleg y plane and three dipole merger $\mathrm{x}$ plane, so the final amplified displacement is not so big. So dogleg x plane and three dipole merger y plane are very sensitive to error. Especially dogleg x plane, special care should be paid, because the displacement inside the merger is very large although it can be cancelled at the exit of merger. 

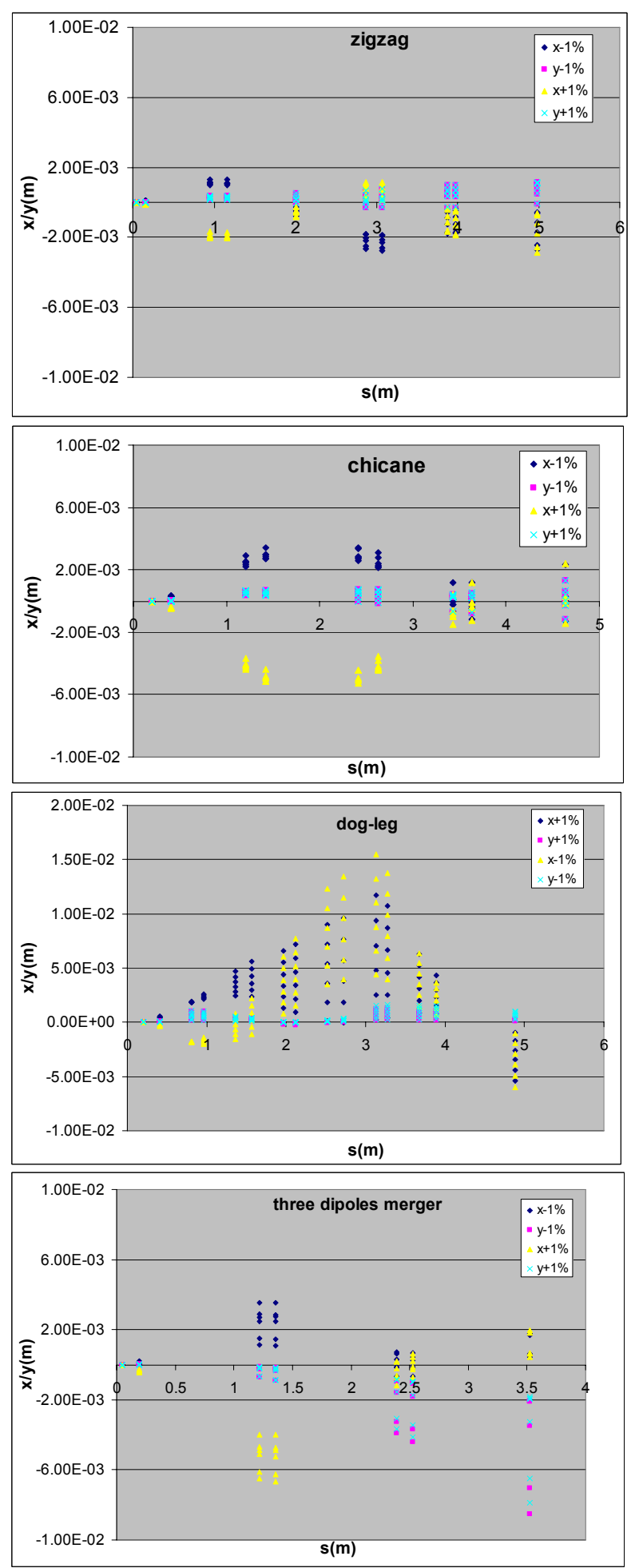

Figure 3: Central orbit displacement at different merger structure study on error sensitivity for $+/-1 \%$ at $5 \mathrm{MeV}$ Note: vertical scale for dogleg is twice of others

With similar method, the longitudinal space charge force changes beam energy, which can be equivalent to the magnet field error in the merger, which is related to the energy spread change. Simulation shows similar results as PARMELA gives[3].
High order term in different merger is also studied on emittance. Generally, it is not a big deal, although chicane and zigzag have smaller high order terms than three dipole merger and dogleg.

These mergers have potential to compress bunch length, with R56 about $0.15 \mathrm{~m}$. With injector providing proper energy-position correlation for $20 \mathrm{pC}$ bunch charge, PARMELA simulation from cathode through merger to the accelerator shows the longitudinal space charge is serious, so that the longitudinal phase space is distorted badly. So it is not a good idea to compress bunch with merger.

\section{SUMMARY}

To maintain beam emittance, different factors at PKU injector are studied. It shows that the RF field gradient is predominant to determine beam emittance, while RF phase and DC voltage has tiny effect. With DIMAD, different merger structure on error sensitivity study shows chicane and zigzag is good. And this method is also applicable to longitudinal space charge force. High order term and possible bunch compression in merger is also studied. It gives a general guidance on the front end design of ERL.

\section{REFERENCE}

[1] Zhu Feng et al, Updated design of the DC-SC photoinjector at Peking University, High power laser and particle beams, 2006(18):653-656

[2] B.E.CARLSTEN, NIM A285, (1989)pp313-319

[3] V.N. Litvinenko, R. Hajima, D. Kayran, NIM A 557, (2006) pp 165-175. 